\title{
Thermal Model Parameter Identification of a Lithium Battery
}

\author{
Dirk Nissing, ${ }^{1}$ Arindam Mahanta, ${ }^{1}$ and Stefan van Sterkenburg ${ }^{2}$ \\ ${ }^{1}$ Faculty of Technology and Bionics, Rhine-Waal University of Applied Sciences, Kleve, Germany \\ ${ }^{2}$ HAN Automotive Research, Hogeschool van Arnhem en Nijmegen, Arnhem, Netherlands \\ Correspondence should be addressed to Dirk Nissing; dirk.nissing@hochschule-rhein-waal.de
}

Received 21 December 2016; Revised 18 May 2017; Accepted 7 June 2017; Published 13 July 2017

Academic Editor: Daniela Proto

Copyright (C) 2017 Dirk Nissing et al. This is an open access article distributed under the Creative Commons Attribution License, which permits unrestricted use, distribution, and reproduction in any medium, provided the original work is properly cited.

\begin{abstract}
The temperature of a Lithium battery cell is important for its performance, efficiency, safety, and capacity and is influenced by the environmental temperature and by the charging and discharging process itself. Battery Management Systems (BMS) take into account this effect. As the temperature at the battery cell is difficult to measure, often the temperature is measured on or nearby the poles of the cell, although the accuracy of predicting the cell temperature with those quantities is limited. Therefore a thermal model of the battery is used in order to calculate and estimate the cell temperature. This paper uses a simple RC-network representation for the thermal model and shows how the thermal parameters are identified using input/output measurements only, where the load current of the battery represents the input while the temperatures at the poles represent the outputs of the measurement. With a single measurement the eight model parameters (thermal resistances, electric contact resistances, and heat capacities) can be determined using the method of least-square. Experimental results show that the simple model with the identified parameters fits very accurately to the measurements.
\end{abstract}

\section{Introduction}

In the last several years the introduction of battery driven vehicles has been significantly increased and will be further increased in the next years. In addition to hybrid electric cars and battery electric cars, e-mobility vehicles such as EBikes (Figure 1), scooters, segway, and hoverboards have been widely introduced [1]. The enabler for those applications is the important battery component where Lithium ion cells provide optimal performance with respect to voltage level, high energy density, low memory effect, high cycle life, and low self-discharge during storage.

It has been shown that the temperature of the battery is important for their performance, efficiency, safety, and capacity [2]. On the one side the temperature is influenced by the environmental temperature; on the other side the temperature of the battery is influenced by the charging and discharging process. As the ageing of the battery cell accelerates when the temperature of a cell is too high, battery management systems (BMS) take into account this effect using the battery temperature. Additionally, with the knowledge of the core temperature a BMS can adapt the current flow so that the efficiency of a battery is at its optimum. Due to the difficulty measuring the battery cell temperature, the temperature is often measured on or near the poles of the cell. In [3] it was shown that the temperature measured at the poles of a dynamically loaded battery varies to the relevant cell temperature. Hence, the accuracy of the measurement at the poles is limited.

Therefore a thermal model of the battery is used in order to calculate the cell temperature. It is the aim to integrate the thermal model into the energy management controller which requires a simple thermal model. Thus, an RC-network representation for the thermal description is used. The difficulty here is to obtain the quantities of equivalent parameters of the RC-network, such as capacities and thermal resistances.

This paper deals with the identification of thermal resistances, heat capacity parameters, and electric contact resistances (ECR) at the battery poles of the thermal model. Instead of measuring and calculating the parameters, the parameters are determined using the identification method of least-square (LS) using input/output measurements only.

The paper is structured as follows: The simplified thermal battery model through an electric RC-network is described in Section 2, followed by the explanation of the test stand and measurement setup in Section 3. The used parameter 


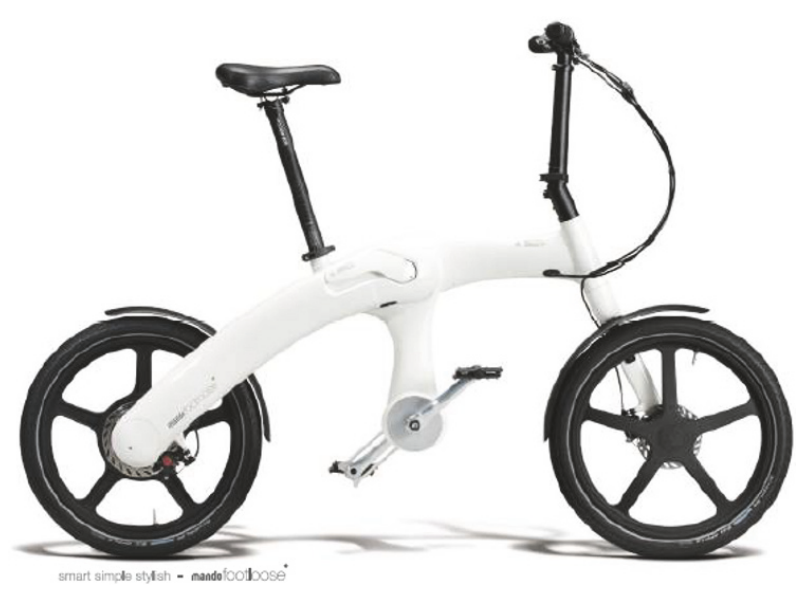

Figure 1: Mando Footloose E-Bike (taken from mando).

identification method is introduced in Section 4. Section 5 describes the application and results of the parameter identification including the validation of the model using independent measurement data. Finally, the results are summarized and some conclusions are drawn in Section 6.

\section{Thermal Battery Model}

With the functional superiority of Lithium batteries over most of its other counterparts, it is undoubtedly a subject of extensive study. Thermal issues with these batteries, like having a high potential for thermal runaway and explosion under high temperature, always threaten the operational safety $[5,6]$. Owing to their narrow operating temperature range, an accurate prediction of battery temperature is absolutely essential so as to maintain the longevity, performance and safety of these Li-ion batteries. Studies [7] have shown that, under typical conditions (such as a HEV drive cycle), the cells may experience a temperature difference between the core and the surface of $10^{\circ} \mathrm{C}$ or more.

Since it is not feasible to measure the temperature of the battery core during runtime, the battery management systems typically use thermal models to predict the core temperature. Existing models range from high fidelity thermal models [8-11] to reduced order models [7, 12-15] capturing the lumped thermal dynamics of the cell. However, the high fidelity models due to their computational complexity are not suitable for onboard application.

2.1. Battery Model. In order to decide the appropriate battery model to be used, observations were made considering the heat conduction inside the core and poles. The transversal heat conductivity of the electroactive region of a cell (the core) is about $0.8[\mathrm{~W} / \mathrm{K} \cdot \mathrm{m}][16]$ and the characteristic length is about $20[\mathrm{~mm}]$, so the heat conduction inside the core is $K_{\text {core }}=40\left[\mathrm{~W} / \mathrm{K} \cdot \mathrm{m}^{2}\right]$. Also for the heat conduction in the $5[\mathrm{~mm}]$ thick polypropylene case of the cell, it is estimated that the heat conduction of the case is $K_{\text {case }}=30\left[\mathrm{~W} / \mathrm{K} \cdot \mathrm{m}^{2}\right]$. This is based on an average heat conductivity of polypropylene of $0.15[\mathrm{~W} / \mathrm{K} \cdot \mathrm{m}]$ [17]. The heat conductance of case to ambient is $6.9\left[\mathrm{~W} / \mathrm{K} \cdot \mathrm{m}^{2}\right]$ as it is measured in the laboratory

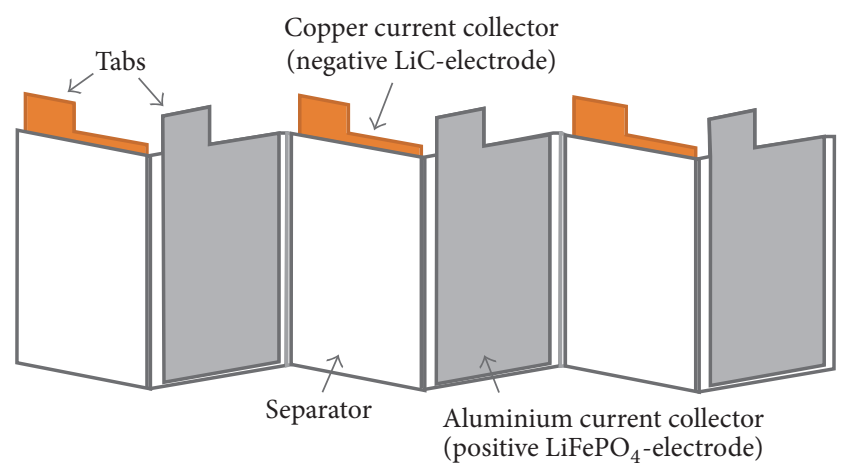

FIGURE 2: Cell core schematic.

(which is a not ventilated room). Also since the article focusses on the temperature of the core and poles, and all heat dissipation occurs in poles and core, and because they have the highest contribution to the total heat capacity of a cell, the Biot number should be calculated as the ratio of heat conduction inside the core to the conduction outside the core. The heat conduction inside the core is $40\left[\mathrm{~W} / \mathrm{K} \cdot \mathrm{m}^{2}\right]$. The heat conduction outside the core is the combined heat transfer through the case and the convection and equals $5.6\left[\mathrm{~W} / \mathrm{K} \cdot \mathrm{m}^{2}\right]$. Thus the Biot-number of 0.14 is considered low enough to assume a lumped thermal model.

For this study the simplified lumped thermal model considered is constructed based on first principles, which incorporates the physics of the various processes occurring in the cell. A 100 [Ah] prismatic Lithium Iron Phosphate cell of Sinopoly (LEP100AHA) [18] was considered for this study. As for prismatic cells, the temperature is often measured on the battery poles; a 1D model is used so as to describe the difference between the temperature at the battery poles and the core. The poles consist of a solid Copper or Aluminium block with an attached cylindrical top for connecting a current cable. The cell construction also implies two Electric contact resistances (ECR) at each pole [3], the ECR from cable to pole and the ECR from pole to current collector sheets. A schematic representation of the construction of the battery core consisting of the current collector sheets [3] is shown in Figure 2. Figure 3 gives the thermal RC representation of the cell model.

However, since not always the internal construction of a battery is well known, a model defined by only three heat capacities, ( 2 poles and the core) and the corresponding thermal resistances is presented. The heat capacities are physical based parameters which are constant and almost time invariant and can be determined by either calculations or experiments. In this contribution the determination of the heat capacities (amongst others) is included in the parameter identification process.

It can be shown that, for a specific heat capacity, the change in temperature $T$ resulting from a net heat flow is given by

$$
C \frac{\mathrm{d} T}{\mathrm{~d} t}=P_{\text {net }}
$$




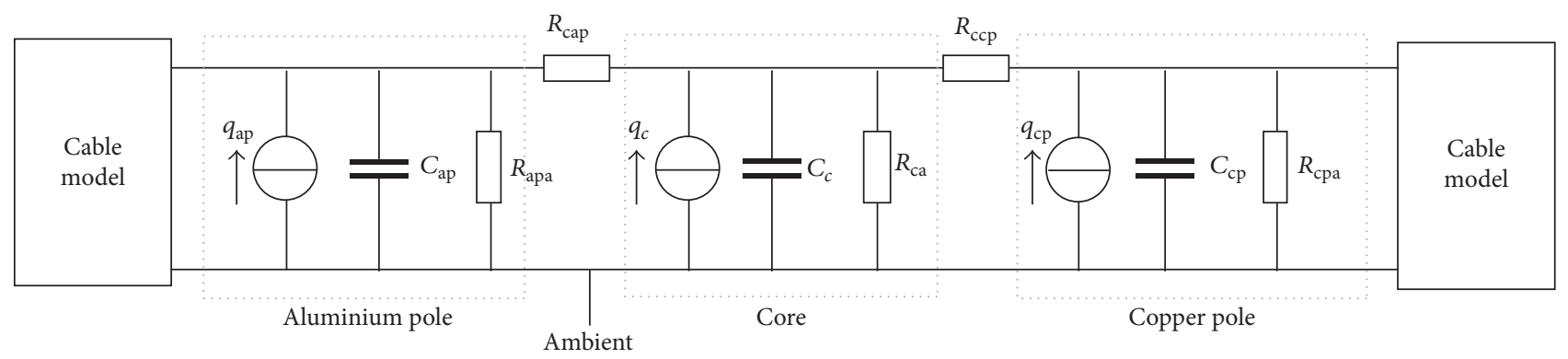

FiguRE 3: Thermal RC-network model of cell.

where $C$ is the heat capacity and $P_{\text {net }}$ the sum of all heat that flows into the heat capacity or is dissipated in the heat capacity. Therefore, for the Copper pole,

$$
C_{\mathrm{cp}} \frac{\mathrm{d} T_{\mathrm{Cu}}}{\mathrm{d} t}=\left(\frac{T_{\mathrm{amb}}-T_{\mathrm{Cu}}}{R_{\text {Cable }}^{\text {th }}}\right)+\left(\frac{T_{\mathrm{Core}}-T_{\mathrm{Cu}}}{R_{\mathrm{ccp}}}\right)+D P_{\mathrm{Cu}} .
$$

Eq. (2) gives the energy balance for the Copper pole with $D P_{\mathrm{Cu}}$ being the heat dissipated at the $\mathrm{Cu}$ pole. The term $\left(T_{\mathrm{amb}}-T_{\mathrm{Cu}}\right) / R_{\mathrm{Cable}}^{\mathrm{th}}{ }^{*}$ represents the heat flow from the surroundings and the connecting cable to the $\mathrm{Cu}$ pole, $R_{\text {Cable }}^{\text {th }}{ }^{*}$ being the equivalent resistance between $R_{\mathrm{th}, \text { Cable }}$ and $R_{\mathrm{cpa}}$. The heat flow from the core to the pole is represented by $\left(T_{\text {Core }}-T_{\mathrm{Cu}}\right) / R_{\text {ccp }}$. Similarly for the Aluminium pole,

$$
C_{\mathrm{ap}} \frac{\mathrm{d} T_{\mathrm{Al}}}{\mathrm{d} t}=\left(\frac{T_{\mathrm{amb}}-T_{\mathrm{Al}}}{R_{\mathrm{Cable}}^{\mathrm{th}}{ }^{*}}\right)+\left(\frac{T_{\mathrm{Core}}-T_{\mathrm{Al}}}{R_{\text {cap }}}\right)+D P_{\mathrm{Al}}
$$

and also for the battery core,

$$
\begin{aligned}
C_{\text {core }} \frac{\mathrm{d} T_{\text {core }}}{\mathrm{d} t}= & \left.\frac{T_{\mathrm{amb}}-T_{\text {core }}}{R_{\text {ca }}}\right)-\left(\frac{T_{\text {Core }}-T_{\mathrm{Cu}}}{R_{\text {ccp }}}\right) \\
& -\left(\frac{T_{\text {core }}-T_{\mathrm{Al}}}{R_{\text {cap }}}\right)+D P_{\text {core }}
\end{aligned}
$$

with $D P_{\text {core }}$ being the heat dissipated at the battery core.

Also essential is the inclusion of the thermal model of the connecting cable that connects the poles to a load. The contribution of the cable can be characterized as a heat conductor between the core and the environment and also as an extra heat capacitance as the ohmic heat dissipated in the cable heats up the pole, which in turn affects the dynamic behavior at the pole. As such the cable model as described by [3] has been used for this study. The effective heat dissipation in the cable is hence given by $q_{\mathrm{sc}}=\left(R_{\mathrm{el} \text {,cable }} * I^{2}\right) /(1+j * \omega *$ $\tau_{\text {cable }}$ ) with $\tau_{\text {cable }}$ as the thermal time constant.

\section{Test Stand and Measurement Setup}

The battery measurement setup is used to measure the electrical and thermal characteristics of a battery pack, which consists of 1 to 12 cells. Figure 4 shows the block scheme of the measurement setup.

The measurable quantities in the Lithium cell are the temperature of the poles and the cell body with the variation of the input load current. For the purpose of measurement, the current profile used was a symmetrical one, with symmetrical charge discharge cycles. With the load current being symmetrical, there is no net electrochemical conversion of energy. Thus all the electrical energy input of the battery is dissipated into heat and can be determined from the measured voltage and current [3]. As such, there were two sets of measurements taken (see Figure 5), the first, set 1 (to investigate the thermal behavior at a somewhat constant load), where the load current is a continuous series of charge discharge cycles with a constant amplitude of 125 [A] and pulse width of 20 [s]. The second set of measurements, set 2 , which were taken to investigate the dynamic behavior of the poles and the core, consisted of 3 subsequent charge/discharge cycles followed by a rest period of 480 [s].

The pulses had a pulse width of 80 [s] and an amplitude of 150 [A].

The series of pulses also guarantee that the relevant eigenfrequencies of the system are stimulated which is essential for a system identification process.

It can be noted, that, for one charge/discharge cycle, the following principles apply:

$$
\overline{P_{\text {cycle }}}=\frac{1}{2}\left(\overline{U_{\text {charge }}}-\overline{U_{\text {discharge }}}\right) \cdot I_{\text {plus }},
$$

where $\overline{P_{\text {cycle }}}$ is the average power dissipation during one charge/discharge cycle, $\overline{U_{\text {charge }}}$ the average cell voltage during the charge pulse, $\overline{U_{\text {discharge }}}$ the average cell voltage during the discharge pulse, and $I_{\text {plus }}$ the magnitude of charge/discharge current. With the two different current load profiles supplied to the battery, the outputs measured shown in Figure 6 were categorized under the following:

Tpole $\mathrm{Cu}_{\mathrm{Cu}}$ is measured temperature at the Copper pole.

Tpole $_{\mathrm{Al}}$ is measured temperature at the Aluminium pole.

$T_{\text {case }}$ is measured temperature at the middle of the case.

Ambient is ambient temperature measured at a distance of $5 \mathrm{~cm}$ from the cell.

\section{Parameter Identification}

Aim of the identification procedure is to determine the substitution parameters of the thermal model of the battery, 


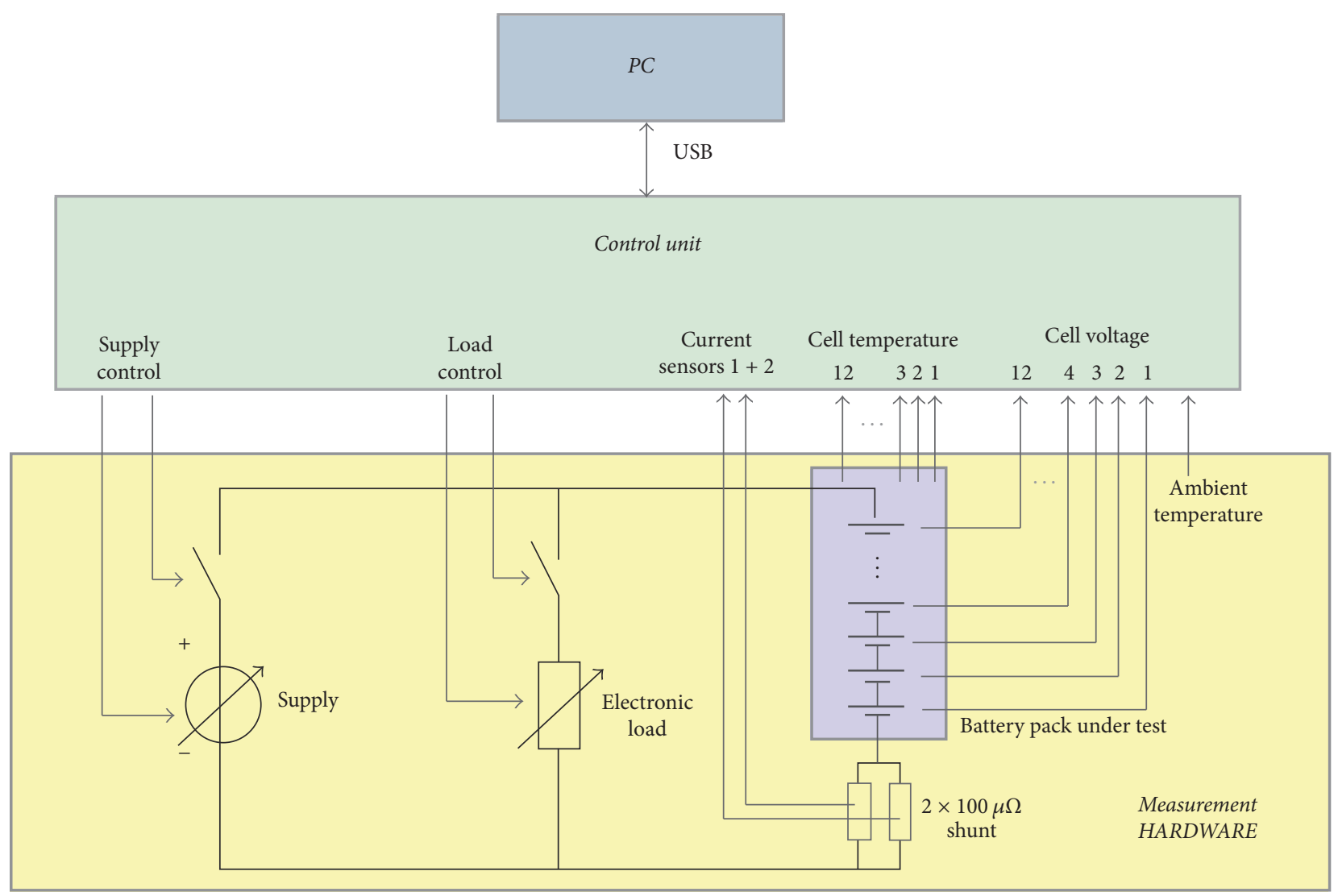

Figure 4: Block scheme of the measurement setup.

based on the input signal $i$ and output signal $T$, where $i$ is the load current profile with a dynamic charge/discharge characteristic and $T$ is the temperature of either the Copper or Aluminium pole. The model parameters are optimized in that the error $e(k)$ between the real process and the model becomes a minimum; see Figure 7. Parameter optimization algorithms have a high degree of development and are widely applied in Toolboxes, such as MATLAB/Simulink.

In order to apply a parameter identification approach the model has to fulfill the following requirements:

(1) Stability of the model; otherwise a measurement is not possible,

(2) The model has to be controllable; otherwise a state cannot be stimulated and hence cannot be identified, and

(3) The model has to be observable; otherwise states cannot be reconstructed from input/output measurements.

The properties of controllability and observability can be easily proved by use of the Kalman criteria [4].

Using the method of least-square (LS) for dynamic systems, the cost function is formulated as

$$
V(\widehat{\boldsymbol{\theta}})=\sum_{k=n}^{n+N} e^{2}(k, \widehat{\boldsymbol{\theta}})=\mathbf{e}(k, \widehat{\boldsymbol{\theta}})^{T} \mathbf{e}(k, \widehat{\boldsymbol{\theta}})
$$

and has to be minimized, where $\mathbf{e}(k, \widehat{\boldsymbol{\theta}})$ is the model error dependent on the coefficients of the difference equation

$$
\widehat{\boldsymbol{\theta}}=\left[\widehat{a}_{1} \cdots \widehat{a}_{n} \mid \widehat{b}_{1} \cdots \widehat{b}_{n}\right]^{T}
$$

which represent the parameters to be estimated. Calculating the derivative of the cost function and setting it to zero, an analytical equation for the estimated parameters can be formulated:

$$
\widehat{\boldsymbol{\theta}}=\left(\boldsymbol{\Psi}^{T} \boldsymbol{\Psi}\right)^{-1} \boldsymbol{\Psi}^{T} \mathbf{y}
$$

where $\Psi$ is the data matrix and contains the input and output measurement data $[4,19]$.

A disadvantage of the model result is the sensitivity against disturbances and model uncertainties. Additionally the model is overdetermined as the thermal battery model contains more coefficients than substitution parameters. Hence an indirect approach is applied [20]. The errors at the time $k$ are stored in an error vector

$$
\mathbf{e}(\widehat{\boldsymbol{\theta}})=[e(1, \widehat{\boldsymbol{\theta}}) \cdots e(N, \widehat{\boldsymbol{\theta}})]^{T}
$$

Similar to the LS-method the quadratic cost function can be formulated as

$$
V(\widehat{\boldsymbol{\theta}})=e(\widehat{\boldsymbol{\theta}})^{T} e(\widehat{\boldsymbol{\theta}})
$$



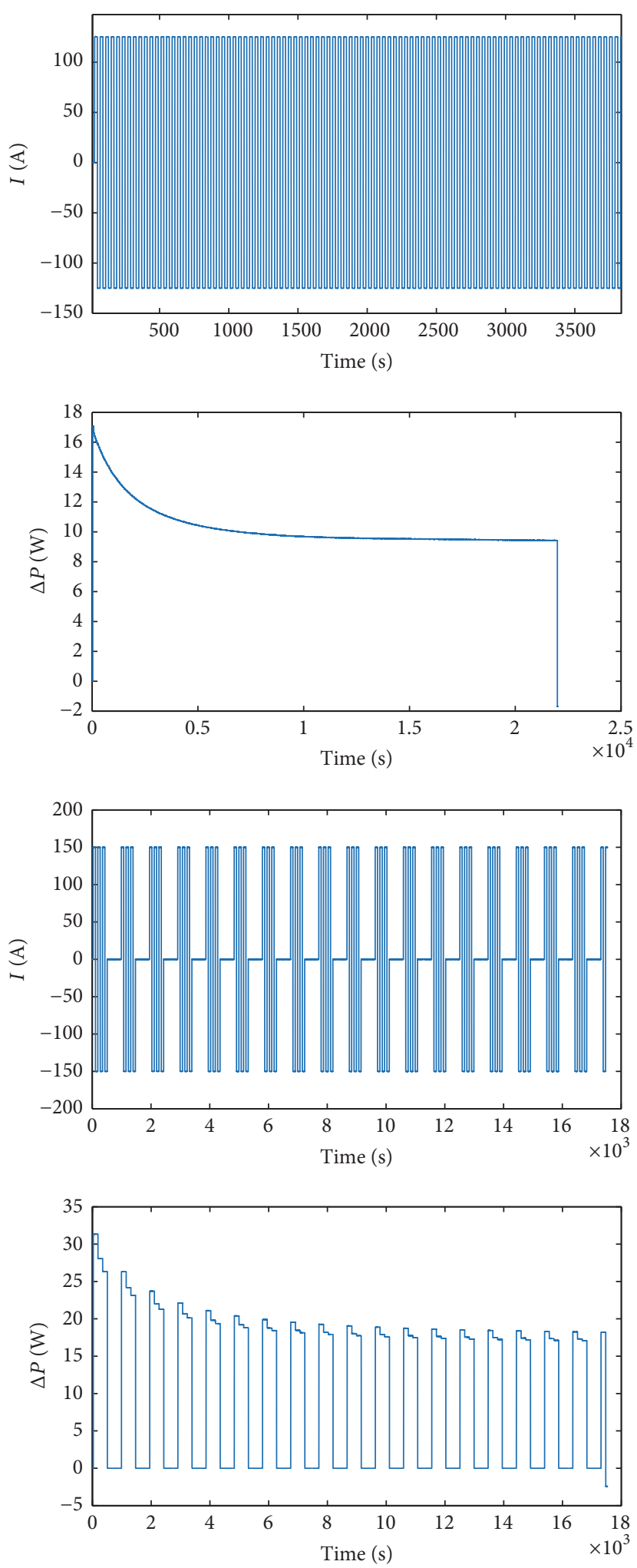

FIGURE 5: Load current and average power dissipation for the two measurement sets $(1,2)$. 

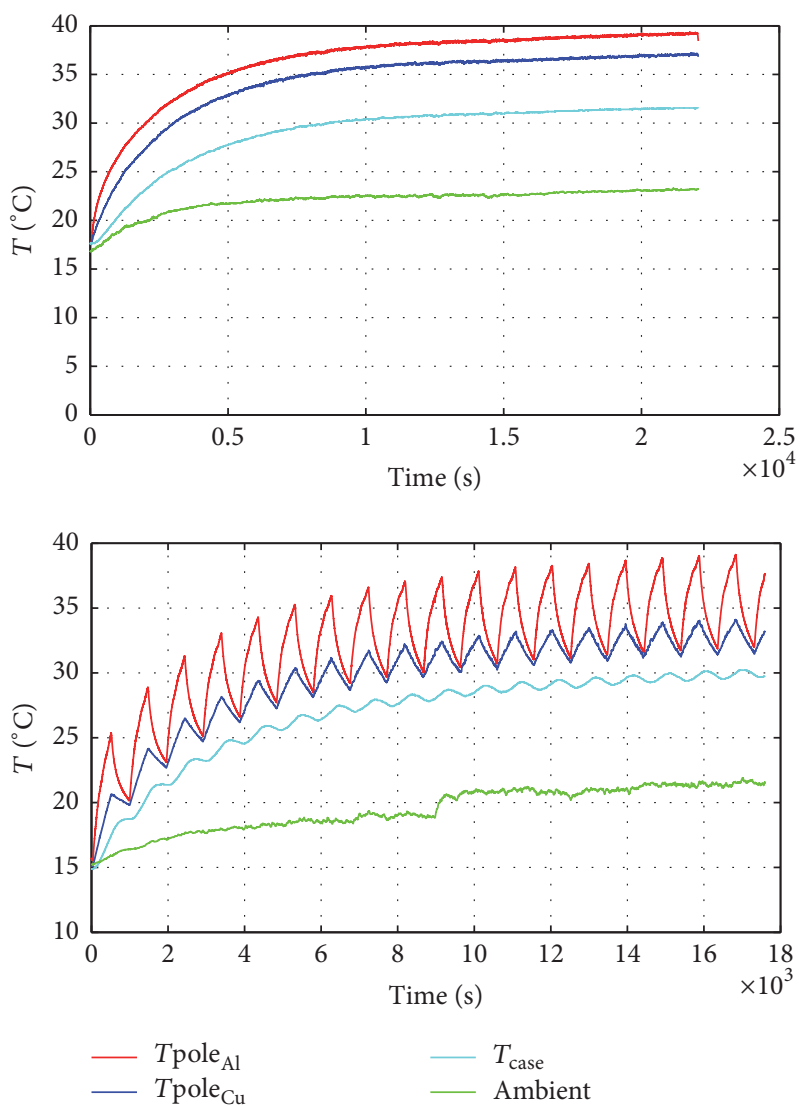

FIGURE 6: Output measurement profiles for measurement sets 1, 2.

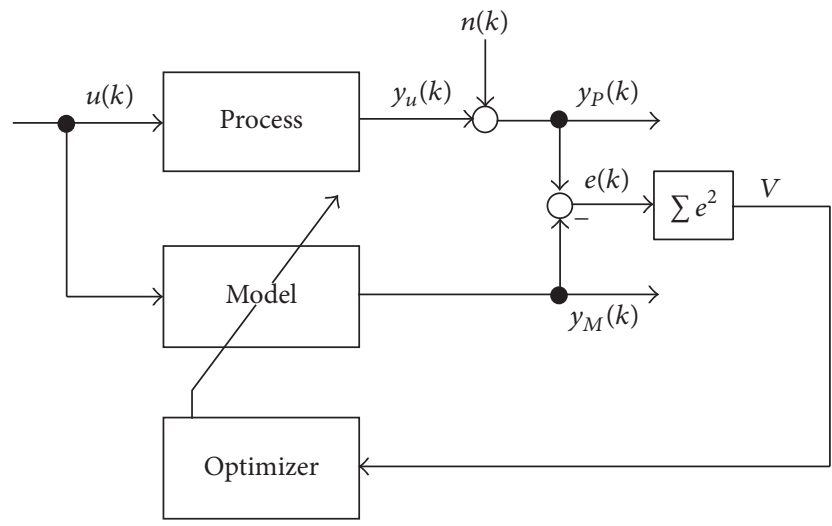

FIGURE 7: Schematic diagram of identification arrangement [4].

and has to be minimal. It yields

$$
\nabla V(\widehat{\boldsymbol{\theta}})=\frac{\partial V(\widehat{\boldsymbol{\theta}})}{\partial \widehat{\boldsymbol{\theta}}}=\left[\frac{\partial V(\widehat{\boldsymbol{\theta}})}{\partial \hat{\theta}_{1}} \cdots \frac{\partial V(\widehat{\boldsymbol{\theta}})}{\partial \hat{\theta}_{M}}\right]=\mathbf{0}^{T} .
$$

Using the Gauß-Newton-approach and the initial condition for the parameters $\widehat{\boldsymbol{\theta}}_{0}$ the estimation is calculated "step by step" through

$$
\widehat{\boldsymbol{\theta}}_{i+1}=\widehat{\boldsymbol{\theta}}_{i}-\mathbf{H}^{-1}\left(\widehat{\boldsymbol{\theta}}_{i}\right) \nabla V\left(\widehat{\boldsymbol{\theta}}_{i}\right),
$$

where

$$
\mathbf{H}(\widehat{\boldsymbol{\theta}})=\frac{\partial^{2} V(\widehat{\boldsymbol{\theta}})}{\partial \widehat{\boldsymbol{\theta}}^{T} \partial \widehat{\boldsymbol{\theta}}}
$$

represents the Hesse-matrix. With the introduction of the Jacobian-matrix

$$
\mathbf{J}(\widehat{\boldsymbol{\theta}})=\frac{\partial \mathbf{e}(\widehat{\boldsymbol{\theta}})}{\partial \widehat{\boldsymbol{\theta}}}
$$

the gradient of the cost function can be formulated as

$$
\nabla V(\widehat{\boldsymbol{\theta}})=2 \mathbf{J}(\widehat{\boldsymbol{\theta}})^{T} e(\widehat{\boldsymbol{\theta}})
$$

and taking into account the approximation of the Hessematrix

$$
\mathbf{H}(\widehat{\boldsymbol{\theta}}) \approx 2 \mathbf{J}(\widehat{\boldsymbol{\theta}})^{T} \mathbf{J}(\widehat{\boldsymbol{\theta}})
$$

it yields

$$
\widehat{\boldsymbol{\theta}}_{i+1}=\widehat{\boldsymbol{\theta}}_{i}-\left[\mathbf{J}_{i}\left(\widehat{\boldsymbol{\theta}}_{i}\right)^{T} \mathbf{J}_{i}\left(\widehat{\boldsymbol{\theta}}_{i}\right)\right]^{-1} \mathbf{J}_{i}\left(\widehat{\boldsymbol{\theta}}_{i}\right)^{T} \mathbf{e}_{i}\left(\widehat{\boldsymbol{\theta}}_{i}\right)
$$

for the estimated model parameters. A set of satisfying parameters $\widehat{\boldsymbol{\theta}}^{*}$ are achieved, if the difference between $\widehat{\boldsymbol{\theta}}_{i}$ and $\widehat{\boldsymbol{\theta}}_{i+1}$ is less than a given threshold $\Delta \boldsymbol{\theta}_{\text {min }}$, thus $\left|\widehat{\boldsymbol{\theta}}_{i}-\widehat{\boldsymbol{\theta}}_{i+1}\right|<$ $\Delta \boldsymbol{\theta}_{\text {min }}$. in [4].

The proof of convergence of the LS-method is formulated

\section{Thermal Model Analysis}

For the model analysis, the theoretical model (Figure 3) was used and the output of the model was compared with the actual process output as in Figure 6 (set 1). For the initial values of the battery parameters, the values experimentally determined by [3] et al. were considered. The model output for the first measurement set using the empirically determined parameters was found out to be quite satisfactory however with an approximate error of 5\% (Figure 8). The deviations for both output measurements have been minimized by iteratively changing the values of the experimentally determined parameters by employing a nonlinear least square method using the Trust-Region-Reflective algorithm; see Section 4 . The new parameter values obtained were found to represent the measurements quite accurately with an approximate deviation of around $0.5-1 \%$ (Figure 9).

The parameters obtained were now validated using a second independent measurement set with 150 [A] maximum current input. The results obtained were satisfactory in terms of the obtained curve fit (Figure 10). The obtained parameters are listed down in Table 1. The estimated parameters being close to the empirically determined ones suggest the overall dimensional correctness of the system model. 


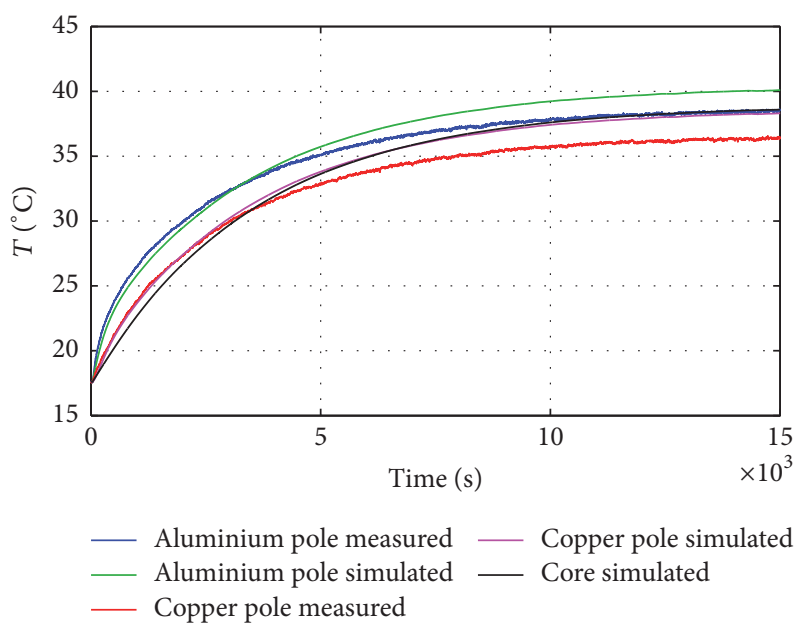

FIGURE 8: Model output and system output comparison for 1st set of measurements.

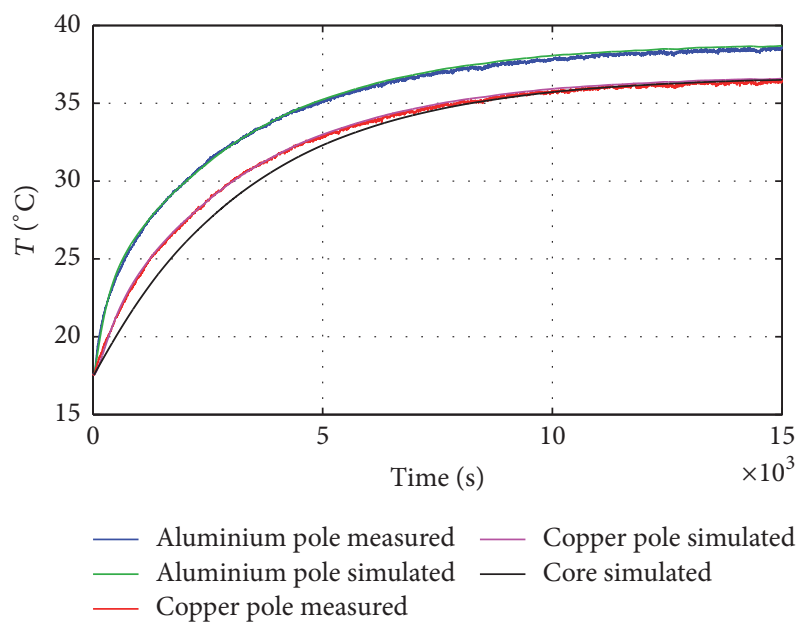

FIGURE 9: Model output and system output comparison for 1st set of measurements with tuned parameters.

\section{Summary and Conclusion}

In this paper a least-square parameter identification method is applied to determine the parameters of a thermal model of a battery cell. In this paper a simplified lumped thermal model is used and described through an equivalent RC-network. As it is difficult to determine the thermal resistances and heat capacities experimentally or empirically, the LS-parameter identification method has been applied based on input- and output measurements. Here the load current of the battery represents the input while the temperatures at the poles represent the outputs of the measurement. Experimental results show that the simple model with the identified parameters fits very accurately to the measurements (less than $1 \%$ deviation) and even the validation shows an adequate behavior, where the dynamic temperature deviation is less than $5 \%$.

The thermal model can be used as a basis for future research and development. For example a model based observer can be applied to predict the core temperature of the

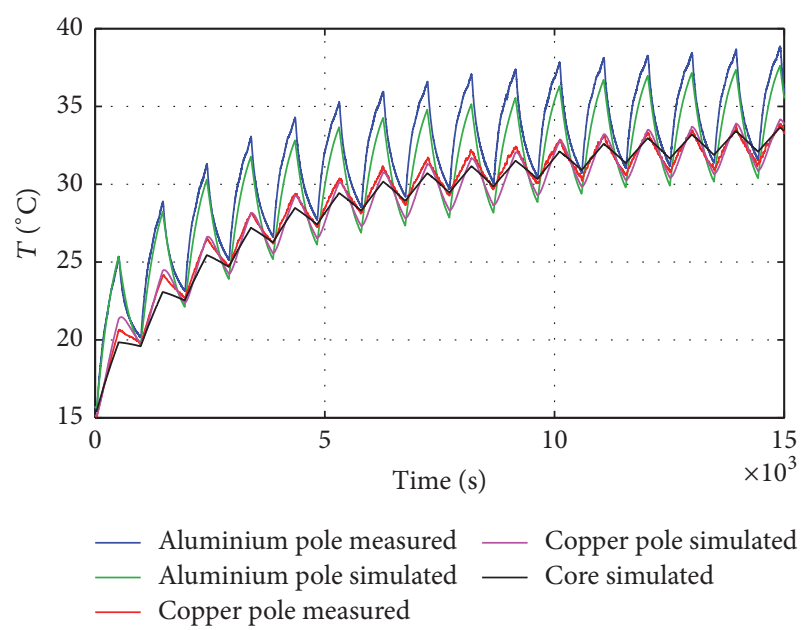

FIGURE 10: Model output and system output comparison for 2 nd set of measurements with the tuned parameters.

TABLE 1: Obtained parameter values.

\begin{tabular}{lcc}
\hline Parameter & Empirically determined & Parameter identification \\
\hline$C_{\mathrm{ap}}\left[\mathrm{JK}^{-1}\right]$ & 53 & 52.997 \\
$C_{c}\left[\mathrm{JK}^{-1}\right]$ & 2900 & 3080.2 \\
$C_{\mathrm{cp}}\left[\mathrm{JK}^{-1}\right]$ & 62 & 84.792 \\
$R_{\mathrm{ca}}\left[\mathrm{KW}^{-1}\right]$ & 1.64 & 1.3731 \\
$R_{\text {cap }}\left[\mathrm{KW}^{-1}\right]$ & 2.2 & 3.5244 \\
$R_{\text {ccp }}\left[\mathrm{KW}^{-1}\right]$ & 1.7 & 3.1336 \\
$E C R_{\mathrm{Al}}[\mu \Omega]$ & 87 & 73.786 \\
$E C R_{\mathrm{Cu}}[\mu \Omega]$ & 21 & 22.207 \\
\hline
\end{tabular}

battery cell. As the model is relatively simple, the observer can be executed on simple microcontrollers in order to improve the battery management of E-Bikes and can be applied to any other battery operated systems with a relatively high power load. Additionally, the information of the battery core temperature can be taken into account for a BMS in order to operate at a maximum/optimal efficiency.

\section{Abbreviations}

\section{Network Components Defined in the Thermal \\ RC-Network Model}

$q_{c}: \quad$ Heat generated in the core (caused by the overvoltage losses and entropic heat exchange)

$q_{\mathrm{ap}}$ : Heat generated at the Aluminium pole caused by the current through the ECR's at the pole

$q_{\mathrm{cp}}$ : Heat generated at the Copper pole caused by the current through the ECR's at the pole

$C_{c}$ : Heat capacity of the core

$C_{\text {ap }}$ : Heat capacity of Aluminium pole

$C_{\mathrm{cp}}$ : Heat capacity of Copper pole

$R_{\text {cap }}$ : Thermal resistance from core to Aluminium pole

$R_{\text {ccp }}$ : Thermal resistance from core to Copper pole 
$R_{\text {apa }}$ : Thermal resistance from Aluminium pole to ambient

$R_{\text {ca }}$ : Thermal resistance from core to ambient

$R_{\text {cpa }}$ : Thermal resistance from Copper pole to ambient

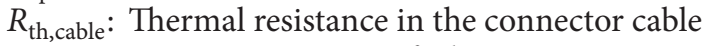

$E C R_{\mathrm{Al}}$ : Contact resistance of Aluminium

$E C R_{\mathrm{Cu}}$ : Contact resistance of Copper

$D P_{\mathrm{Cu}}$ : Heat dissipated at the Copper pole

$D P_{\mathrm{Al}}$ : Heat dissipated at the Aluminium pole

$D P_{\text {Core }}$ : Heat dissipated at the cell core.

\section{Conflicts of Interest}

The authors declare that there are no conflicts of interest regarding the publication of this paper.

\section{References}

[1] G. Pistoia, Battery Operated Devices and Systems, Elsevier, Oxford, UK, 2009.

[2] T. Waldmann, M. Wilka, M. Kasper, M. Fleischhammer, and M. Wohlfahrt-Mehrens, "Temperature dependent ageing mechanisms in Lithium-ion batteries-a Post-Mortem study," Journal of Power Sources, vol. 262, pp. 129-135, 2014.

[3] S. van Sterkenburg and B. Veenhuizen, "The modelling of the temperature at the poles and core of a large prismatic LiFePO4 cells," in Proceedings of the European Battery, Hybrid and Fuel Cell Electric Vehicle Congress, Brussels, Belgium, 2015.

[4] R. Isermann and M. Münchhof, Identification of Dynamic Systems, Springer, Berlin, Germany, 2011.

[5] Q. Wang, P. Ping, X. Zhao, G. Chu, J. Sun, and C. Chen, "Thermal runaway caused fire and explosion of lithium ion battery," Journal of Power Sources, vol. 208, pp. 210-224, 2012.

[6] T. M. Bandhauer, S. Garimella, and T. F. Fuller, "A critical review of thermal issues in lithium-ion batteries," Journal of the Electrochemical Society, vol. 158, no. 3, pp. R1-R25, 2011.

[7] C. Forgez, D. Vinh Do, G. Friedrich, M. Morcrette, and C. Delacourt, "Thermal modeling of a cylindrical $\mathrm{LiFePO}_{4}$ /graphite lithium-ion battery," Journal of Power Sources, vol. 195, no. 9, pp. 2961-2968, 2010.

[8] C. Y. Wang and V. Srinivasan, "Computational battery dynamics (CBD) - electrochemical/thermal coupled modeling and multiscale modeling," Journal of Power Sources, vol. 110, no. 2, pp. 364-376, 2002.

[9] NASA Technical Reports Server (NTRS), "Computational fluid dynamics modeling of nickel hydrogen batteries," Tech. Rep. 20000112900, 2000.

[10] A. Pruteanu, B. V. Florean, G. M. Moraru, and R. C. Ciobanu, "Development of a thermal simulation and testing model for a superior lithium-ion-polymer battery," in Proceedings of the 13th International Conference on Optimization of Electrical and Electronic Equipment, OPTIM, pp. 947-952, IEEE, Brasov, Romania, May 2012.

[11] Y. Kim, J. B. Siegel, and A. G. Stefanopoulou, "A computationally efficient thermal model of cylindrical battery cells for the estimation of radially distributed temperatures," in Proceedings of the American Control Conference, ACC, pp. 698-703, IEEE, Washington, DC, USA, June 2013.

[12] D. Bernardi, E. Pawlikowski, and J. Newman, "A general energy balance for battery systems," Journal of the Electrochemical Society, vol. 132, no. 1, pp. 5-12, 1985.
[13] A. Smyshlyaev, M. Krstic, N. Chaturvedi, J. Ahmed, and A. Kojic, "PDE model for thermal dynamics of a large Li-ion battery pack," in Proceedings of the American Control Conference, ACC, pp. 959-964, IEEE, San Francisco, CA, USA, July 2011.

[14] R. R. Richardson and D. A. Howey, "Sensorless battery internal temperature estimation using a Kalman filter with impedance measurement," IEEE Transactions on Sustainable Energy, vol. 6, no. 4, pp. 1190-1199, 2015.

[15] X. Lin, H. E. Perez, J. B. Siegel et al., “Online parameterization of lumped thermal dynamics in cylindrical lithium ion batteries for core temperature estimation and health monitoring," IEEE Transactions on Control Systems Technology, vol. 21, no. 5, pp. 1745-1755, 2013.

[16] M. Guo, G.-H. Kim, and R. E. White, "A three-dimensional multi-physics model for a Li-ion battery," Journal of Power Sources, vol. 240, pp. 80-94, 2013.

[17] D. Tripathi, Practical Guide to Polypropylene, iSmithers Rapra Publishing, 2002.

[18] S. van Sterkenburg, T. Fleuren, B. Veenhuizen, and J. Groenewegen, "Design and test of a battery pack simulator," in Proceedings of the EVS27 International Battery, Hybrid and Fuel Cell Electric Vehicle Symposium, IEEE, Barcelona, Spain, November 2013.

[19] L. Ljung, System Identification: Theory for the User, Prentice Hall, Upper Saddle River, NJ, USA, 1999.

[20] D. Nissing and J. Polzer, "Parameter identification of a substitution model for a flexible link," in Proceedings of the IFAC Symposium on System Identification, Santa Barbara, Calif, USA, 2000. 


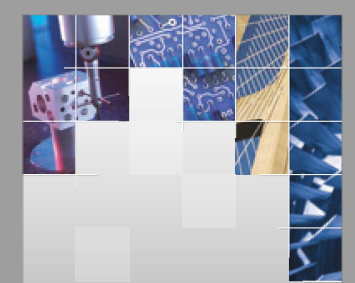

\section{Enfincering}
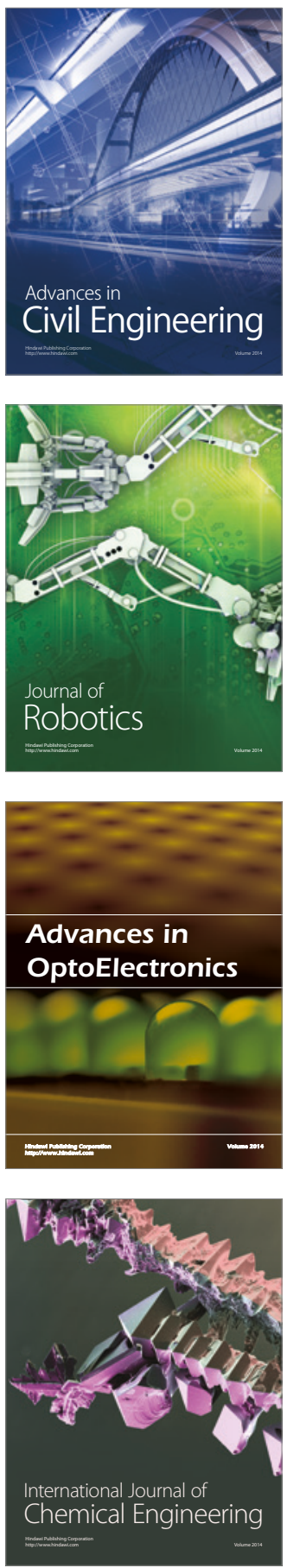

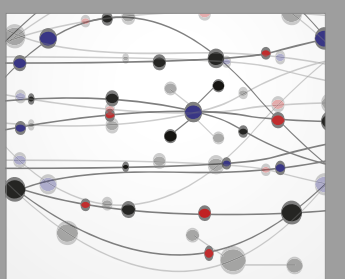

The Scientific World Journal

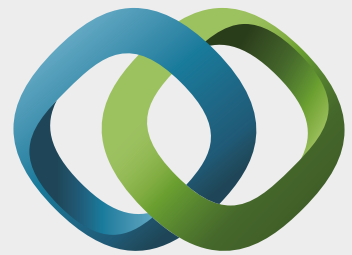

\section{Hindawi}

Submit your manuscripts at

https://www.hindawi.com
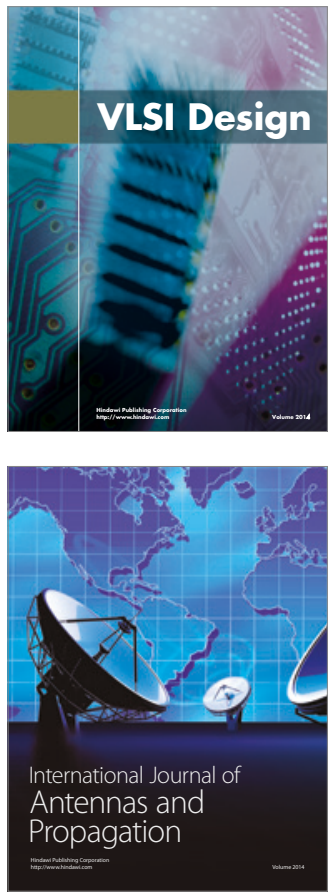

\section{Rotating}

Machinery
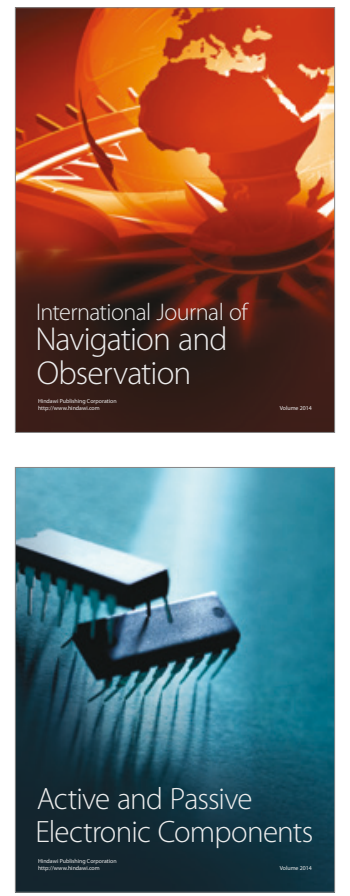
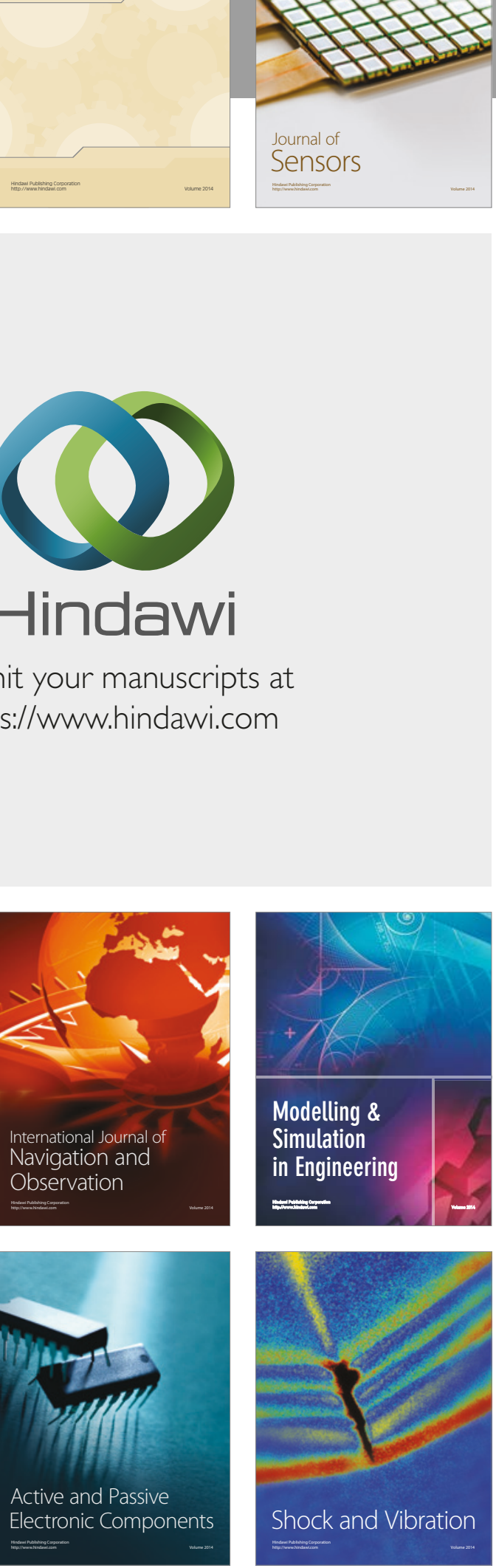
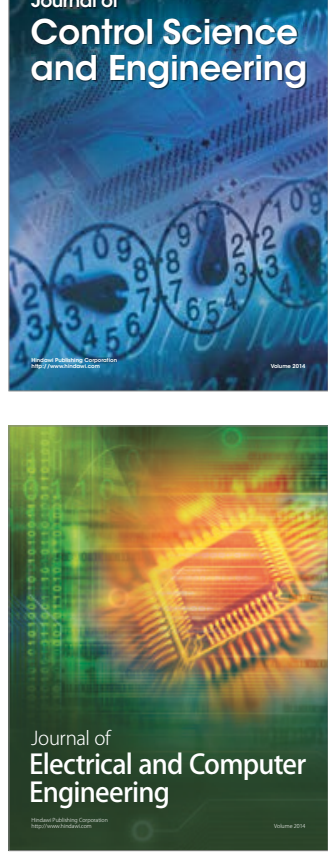

Distributed

Journal of

Control Science

and Engineering
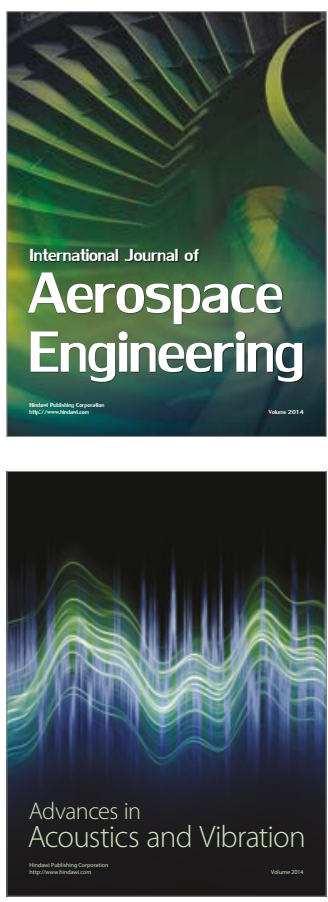

Sensor Networks 